\title{
High-dose-rate interstitial brachytherapy for mobile tongue cancer: preliminary results of a dose reduction trial
}

\author{
Hironori Akiyama, DDS, PhD!, Ken Yoshida, MD, PhD², Hideya Yamazaki, MD, PhD³, Tadashi Takenaka, RTT4 \\ Tadayuki Kotsuma, MD, PhD 5 , Koji Masui, MD³, Yasuo Yoshioka, MD, PhD ${ }^{6}$, Takumi Arika, DDS, PhD", \\ Kimishige Shimizutani, DDS, PhD', Eiichi Tanaka, MD, PhD 5 \\ 'Department of Oral Radiology, Osaka Dental University, ${ }^{2}$ Department of Radiology. Osaka Medical College, ${ }^{3}$ Department of Radiology. \\ Kyoto Prefectural University of Medicine, ${ }^{4}$ Department of Radiology. National Hospital Organization Osaka National Hospital, \\ ${ }^{5}$ Department of Radiation Oncology, National Hospital Organization Osaka National Hospital, 'bepartment of Radiation Oncology. \\ Osaka University Graduate School of Medicine, ${ }^{7}$ Department of Oral Surgery, National Hospital Organization Osaka National Hospital, Japan
}

\begin{abstract}
Purpose: To compare the outcome of our facility with another about the shortened schedule (60 Gy in 10 fractions to 54 Gy in 9 fractions) of high-dose-rate interstitial brachytherapy (HDR ISBT) for mobile tongue cancer.

Material and methods: Eighteen patients were treated with HDR ISBT as a monotherapy in dose reduction schedule with some unique technique to determine the border of tumor accuracy (lugol's staining and metal marker), and to minimize adverse effect (lead-lined silicon block) at our facility.

Results: The 2-year local and regional control rates and cause-specific survival rate were $82 \%, 80 \%$, and $83 \%$ and moderate to severe late complications occurred in five patients $(28 \%)$, which were almost the same treatment results achieved by another facility.

Conclusions: We recommend $54 \mathrm{~Gy}$ in 9 fractions over 7 days as a feasible treatment to reduce patient discomfort in mobile tongue cancer patients.

Key words: dose reduction, high-dose-rate brachytherapy, tongue cancer.

\section{Purpose}

Mobile tongue cancer is highly curable with radiation therapy, especially interstitial brachytherapy (ISBT). Many institutions have reported successful results using low-dose-rate (LDR) ISBT [1-5]. However, there are some shortcomings, such as radiation exposure to medical staff and no dose optimization, with this treatment. To solve these problems, some institutions adopted high-dose-rate (HDR) ISBT using a remote afterloading system. There are some advantages of HDR ISBT; for example, no radiation exposure to medical staff and better dose optimization after implantation. For HDR ISBT treatment, they implemented a dose fractionation schedule of $60 \mathrm{~Gy}$ in 10 fractions over approximately 8 days at a distance of $5 \mathrm{~mm}$ from the radioactive source and achieved good treatment results [6], which were comparable to those of LDR ISBT [6-9]. However, HDR ISBT is uncomfortable and poses a risk to patients, as maintaining applicators in the oral to submandibular region for long periods causes significant irritation and a risk of sputum aspiration.
Thus, it is necessary to shorten the treatment period, even if it is by only one day. In addition, a recommended dose fractionation schedule has not yet been adopted by the Groupe Européen de Curiethérapie-European Society for Therapeutic Radiology and Oncology (GEC-ESTRO) or the American Brachytherapy Society $[10,11]$. Some authors investigated alternative treatment schedules conducted at Osaka University Hospital to reduce the dose amount and treatment period without compromising treatment outcomes, and determined that 54 Gy in 9 fractions showed outcomes compatible to $60 \mathrm{~Gy}$ in 10 fractions [12,13]. In 2001, we adopted this shortened schedule at our institution to affirm the effectiveness of this dose fractionation schedule. In this study, we present the preliminary outcome of HDR ISBT for mobile tongue cancer using the new dose reduced protocol at our institution.

\section{Material and methods}

Between February 2001 and February 2009, 18 patients with previously untreated, locally limited mobile tongue

\footnotetext{
Address for correspondence: Hironori Akiyama, DDS, PhD, Department of Oral Radiology, Osaka Dental Received: 10.11 .2013 University, 1-5-17 Otemae, Chuo-ku, Osaka-City, Osaka, 540-0008, Japan, phone: +81-6-6910-1074, Accepted: 01.02.2014 fax: +81-6-6910-1075, e-mail: akiyama@cc.osaka-dent.ac.jp

Published: 28.03.2014
} 
cancer were treated with HDR ISBT as a monotherapy at our institution. Prior to the treatment, we obtained verbal and written informed consent from all patients. All tumors were histologically identified as squamous cell carcinomas. Table 1 lists patient characteristics. The median patient age was 61 years (range 34-84 years), and median follow-up time was 49 months (range 10-121 months). Using the 2002 Union for International Cancer Control classification system, 3, 11, and 4 patients were classified as T1, T2, and T3, respectively. In regard to morphological type, 8 and 10 patients had superficial - and infiltrative type of cancer, respectively. Implantation was performed under general anesthesia in all patients except one, who received local anesthesia because of insufficient pulmonary function. Inspection and palpation was performed by more than two physicians and Lugol's iodine staining was performed to estimate the superficial extension of the tumor [14-16]. Intraoral ultrasonography (SSD-1000 ${ }^{\circledR}$; Hitachi Aloka Medical Ltd., Tokyo, Japan) was performed to define tumor thickness [17]. Before implantation, at least four titanium seed markers were injected at the anterior, posterior, lateral, and medial edges of the lesion to plan the treatment (Fig. 1) [18]. Two or three planes were adopted for implantation with a custom - made vinyl template for guidance. We selected two planes for implantation if the thickness of the tumor was $\leq 10 \mathrm{~mm}$, and three planes for implantation if the thickness was $>10 \mathrm{~mm}$. As the first step of applicator implantation, an open-ended hard metal guiding needle (Bevel point needle ${ }^{\circledR}$; Elekta AB, Stockholm, Sweden) was inserted from the submandibular region to the mouth floor or tongue mucosa with the guidance of the custom - made vinyl template or intraoral ultrasonography. The needle exit points were determined using the custom - made vinyl template on the tongue surface. The applicators in the lateral plane penetrated the mouth floor mucosa and made direct contact with the lateral border of the tongue mucosa in almost all cases. The applicators in the medial plane were implanted into the edge of tumor lesion. After implantation of the guiding needle, the flexible applicator was replaced. These applicators were then fixed with a but-

Table 1. Clinical characteristics of patients

\begin{tabular}{lc} 
& $N$ \\
\hline Age (years) & $34-84$ \\
\hline Range & 61 \\
\hline Median & 10 \\
\hline Gender & 8 \\
\hline Male & 3 \\
\hline Female & 11 \\
\hline TNM classification & 4 \\
\hline T1N0M0 & 8 \\
\hline T2N0M0 & 10
\end{tabular}

ton in the submandibular region following removal of the guide needle. Two types of flexible applicators were used: one was a linearly implanted Single-leader applicator ${ }^{\circledR}$ (Elekta AB, Stockholm, Sweden) with a one-sided button, whereas the second was a loop Double-leader applicator ${ }^{\circledR}$ (Elekta AB, Stockholm, Sweden). The loop technique was used near the oropharyngeal region because the button of the single-leader applicator on the mucosal surface of the tongue caused irritation for patients (Fig. 1). On the basis of the lesion size, this procedure was repeated to place a sufficient number of flexible applicators. After implantation, the parallelism of the applicators was verified via intraoral ultrasonography (Hitachi Aloka Medical Ltd, Japan).

We performed two dimensional treatment plan. Two orthogonal X-ray images were captured to confirm applicator positions using dummy sources. The basal dose points were defined on central plane. For triangular arrangement of applicators, the basal dose rates were calculated at the center of gravity of each triangle. The reference isodose, where we prescribed $54 \mathrm{~Gy}$ in 9 fractions over 7 days, was $85 \%$ of the mean basal dose rates (Fig. 1). All treatment planning was performed using the PLATO ${ }^{\circledR}$ cancer treatment planning system (Elekta AB, Stockholm,

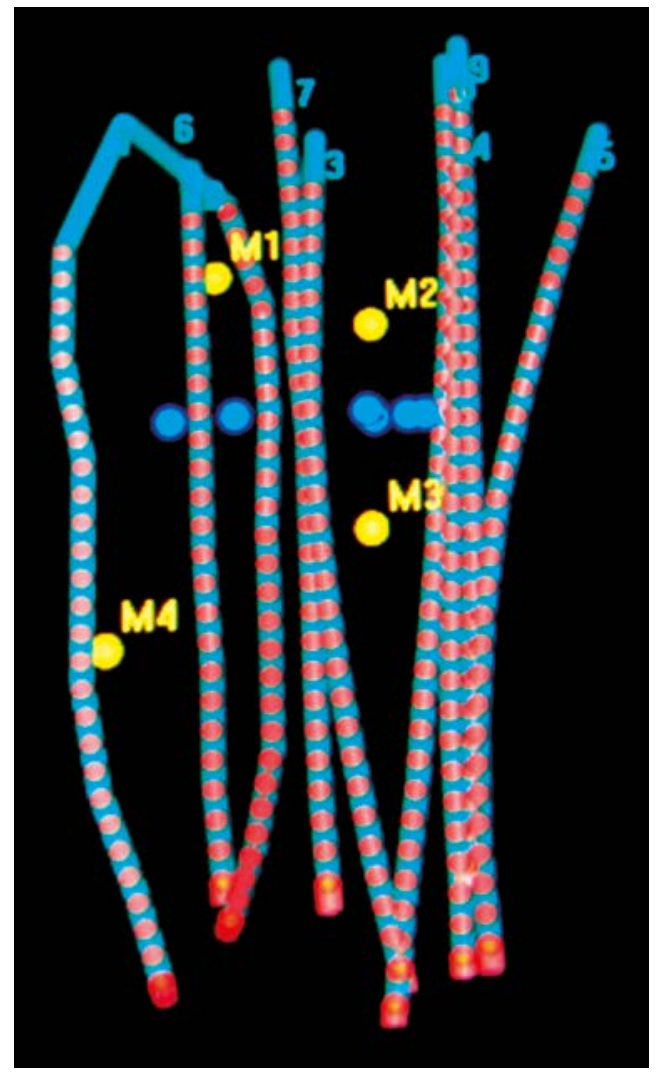

Fig. 1. Four titanium seed markers were injected into the anterior, posterior, lateral, and medial edges of the lesion to plan treatment (yellow points; M1-4). The loop technique was used between applicator number 1 and 6 . The other applicators (numbers 2-5 and 7-9) were linearly implanted. Basal dose points and source dwell points are indicated by blue and red points, respectively 

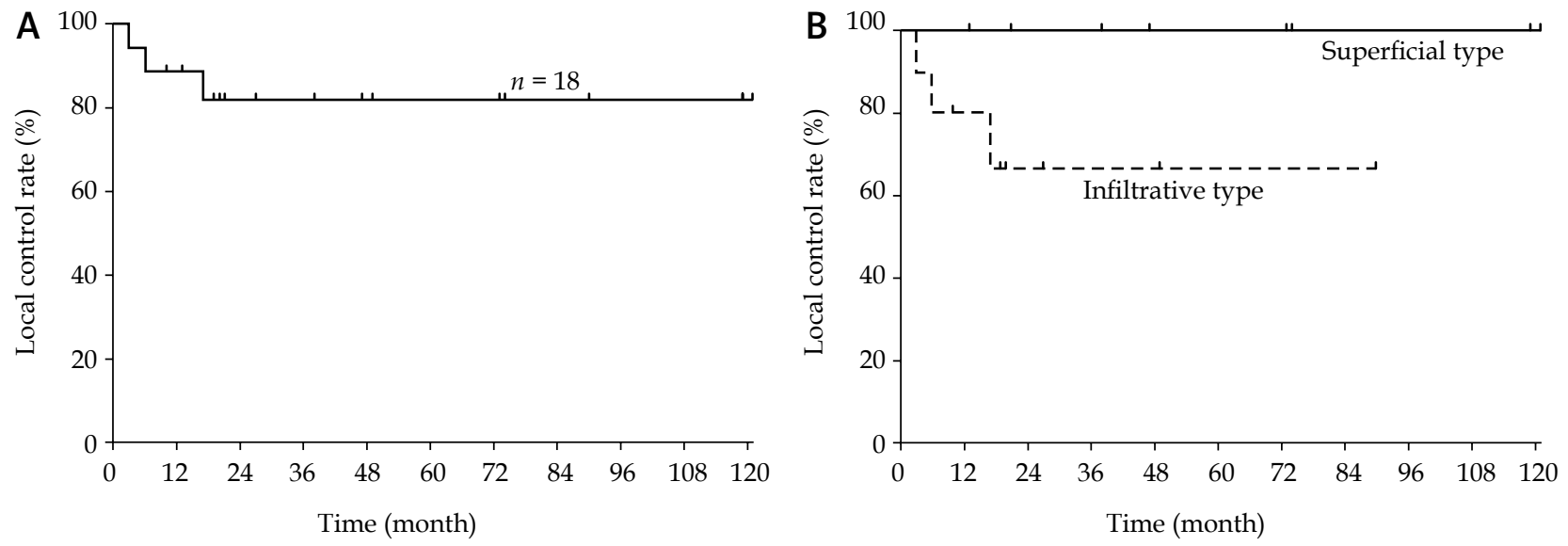

Fig. 2. Local control rate (A) and morphological type (B) in mobile tongue cancer following HDR ISBT

Sweden) with manual modifications [18]. Initially, we used computer optimization (geometrical optimization) then manually modified the dwelling times to deliver prescribed dose to titanium seed markers.

We used a lead block covered with siliconized rubber as a spacer to separate the gingival mucosa and mandible from the radioactive source. Only the silicone block was used to prevent metal artifacts at the time of CT for treatment planning. Two fractions were administered each day, and the time interval between fractions was $>6 \mathrm{~h}$. We used the microSelectron-HDR ${ }^{\circledR}$ brachytherapy applicator (Elekta AB, Stockholm, Sweden) as the ${ }^{192}$ Ir radioactive source. Local and regional control rates, cause-specific survival (CSS) rates, and complications were analyzed. Survival curves were constructed using the Kaplan-Meier method.

\section{Results}

Of all 18 patients, three $\mathrm{T} 2$ cases experienced local recurrence: the first occurred at 3 months after treatment, the second at 6 months, and the third at 17 months. Recurrence in one patient happened because of over - insertion of the siliconized rubber - coated lead block. Two of the three patients underwent salvage surgery, and have maintained local control at the time of this report. There were no recurrences 2 years after treatment. The 2-year local control rate was $82 \%$, whereas the 2-year local control rates were $100 \%, 72 \%$, and $100 \%$ for $\mathrm{T} 1, \mathrm{~T} 2$, and $\mathrm{T} 3$ cases, respectively. The 2-year control rates were $100 \%$, and $67 \%$ for the superficial and infiltrative morphological types, respectively (Fig. 2). The overall neck metastasis occurred in 10 patients. Nine of 10 patients $(90 \%)$ had a lymph node metastasis within 2 years. One patient experienced lymph node metastasis at 34 months after treatment. Of all patients with nodal metastasis, they were treated with radical neck dissection (RND). Two of the 10 patients treated with RND died of nodal metastasis, and 2 with intercurrent disease. Ultimately, nodal metastasis were controlled in 8 of 10 patients $(80 \%)$. For T1, T2, and T3 patients, the neck metastases were occurred in 1 (33\%), 6 (55\%), and 2 (50\%) patients within 2 years after HDR ISBT. In early (T1T2N0) and advanced (T3N0) stages, seven (50\%) and 2 (50\%) pa- tients had a lymph node metastasis, and for the superficial and infiltrative morphological types, two (25\%) and seven (70\%) patients had a nodal metastasis within 2 years after HDR ISBT, respectively. The 2-year CSS rates was $83 \%$. The 2-year CSS rates were $67 \%, 90 \%$, and $75 \%$ for $\mathrm{T} 1, \mathrm{~T} 2$, and $\mathrm{T} 3$ cases, respectively. In early (T1T2N0) and advanced (T3N0) stages, the 2 -year CSS rates were $85 \%$, and $75 \%$, respectively. The 2 -year CSS rates were $88 \%$, and $79 \%$ for the superficial and infiltrative morphological types, respectively.

Concerning adverse effects, such as one severe acute complication of sputum aspiration, which occurred during the treatment period, was transiently managed using a respirator. Moderate to severe late complications occurred in five patients $(28 \%)$ who developed soft tissue necrosis or mandibular complications. One of these five patients received partial sequestrectomy, and all other symptoms were improved via drug and/or hyperbaric oxygen therapy.

\section{Discussion}

To overcome the deficiencies of LDR ISBT for mobile tongue cancer, some institutions have implemented HDR ISBT, but at the outset, lower local control rates were reported in some studies. For example, Lau et al. reported a local control rate of 53\%, using 45.5 Gy in 7 fractions [19]. However, we consider that their dose was insufficient to accomplish a radical curative effect for mobile tongue cancer. In contrast, another institution carried out a Phase I/II study of HDR ISBT for head and neck cancer, and chose a total dose of 60 Gy in 10 fractions over approximately 8 days as a standard schedule for radical HDR ISBT of head and neck cancer [20]. In addition, they conducted a Phase III study to compare the treatment results of HDR ISBT vs. LDR ISBT for mobile tongue cancer. The preliminary and far longer follow-up results of these studies were previously reported $(87 \%$ and $88 \%$ for 5 -year local control and CSS rates, respectively) $[6,7,21]$. In addition, they performed further studies to evaluate their previous results $[8,9]$, and found that the results of HDR ISBT were the same as LDR ISBT. Thus, they concluded that HDR ISBT is a suitable alternative to LDR ISBT for mobile tongue cancer. 
However, we considered that an adequate dose fractionation schedule has not yet been reached. In a previous dose reduction study at Osaka University Hospital, Okamoto et al. [12] reported 3-year local control rates of $90 \%$ and $89.4 \%$ with a total dose of $60 \mathrm{~Gy}$ and $<60 \mathrm{~Gy}$, respectively, whereas Akiyama et al. [13] reported 2- and 3 -year local control rates of $88 \%$ using both $54 \mathrm{~Gy}$ and 60 Gy, respectively. Nevertheless, further studies are needed to evaluate these results. The aim of this study was to confirm the outcome with the shortened schedule of $54 \mathrm{~Gy}$ in 9 fractions over 7 days at our institution. Inoue et al. [6,7] reported that 2-year local control rates of T1T2N0 was $100 \%$, whereas the results in our study of 2-year local control rates, including T1T2N0 cases, was $77 \%$, which were slightly lower than those previously reported. Local control for advanced stage cases was satisfactory. Kakimoto et al. [8] reported that the 2-year local control rate for T3N0-2 was 71\%, whereas that of our study was $100 \%$. The 2 -year control rates were $100 \%$ and $67 \%$ for the superficial and infiltrative morphological types, respectively, which were better than those reported previously [3]. Two of the three patients underwent salvage surgery and have maintained local control at the time of this report. Yamazaki et al. [22] reported a higher ratio of nodal involvement in infiltrative types than in superficial types and our results were consistent with this. Inoue et al. [6,7] reported 2-year nodal control rates for T1T2N0 of 79\%. Shibuya et al. [3] reported that T1T2N0 patients having superficial - type tumors had a lower incidence of a metastasis $(30 \%)$ compared with those having infiltrative - type tumors (54\%). The result of our study showed fifty percent of patients in T1T2N0 cases had a nodal failure, which was slightly higher than those previously reported. Some authors reported that locoregional control rates in the management of early or superficial mobile tongue cancer were higher in the group treated with brachytherapy alone than in that of external irradiation combined with brachytherapy [23-25]. Therefore, we treated our patients with brachytherapy alone. However, our results of nodal failure were worse, because we believe, a larger number of our patients had a infiltrative - type of tumors. In 2009, GEC-ESTRO reported that brachytherapy alone is recommended for T1N0 and T2N0 tumor that are smaller than $4 \mathrm{~cm}$. For tumors $>3-4 \mathrm{~cm}$ or N1 lesions, brachytherapy can be delivered as a boost after 40-45 Gy of external beam irradiation to the neck and oral cavity [10]. Because of this, primary HDR ISBT as monotherapy for the management of patients with mobile tongue cancer might be an option only in selected cases (for example, T1T2N0, superficial tumors). In case of large and infiltrating tumors, external beam irradiation should be added to avoid unacceptably high rate of nodal failure. Of all patients with nodal metastasis, they were treated with RND. Ultimately, $80 \%$ of patients with nodal metastasis were controlled. Thus, we consider that our results were, to some degree, valid.

In the present study, the 2-year CSS rate was $85 \%$. Kakimoto et al. [8] reported 2 - CSS rates of 57\% in T3N0-2 patients, whereas in our study, the 2-year CSS rate was $75 \%$ in T3N0 patients. Thus, we consider that our results were almost similar to those in previous reports.
Inoue et al. [7] and Yamazaki et al. [9] reported that the incidence of complications were $12 \%$ and $10 \%$, respectively. In the present study, the complication rate was $28 \%$; however, the conditions of four patients improved via drug and/or hyperbaric oxygen therapy.

To improve treatment results, we implemented several unique techniques. For example, we applied Lugol's iodine staining [14-16] and titanium seed marker implantation [18] to estimate the extension of the tumor (Fig. 1). In future trials, to decrease the incidence of complications, we will deliver adequate doses to cover the clinical target volume (CTV) without exposing the tongue mucosa and mandible to excessive radiation using this 3 dimensional (3D) image-based treatment planning system. Recently, gynecological planning for HDR ISBT is shifting to 3D image-based planning using computed tomography and magnetic resonance imaging [26]. However, few studies have been reported regarding 3D image-based techniques for head and neck cancer $[27,28]$. We will gradually implement 3D image-based HDR ISBT for mobile tongue cancer using this method at our institution, and we believe that we will achieve better treatment results and reduce complications and patient discomfort.

\section{Conclusions}

In conclusion, we found that a dose reduction to $54 \mathrm{~Gy}$ in 9 fractions over 7 days in HDR ISBT for mobile tongue cancer is feasible, especially for T1T2N0, superficial tumors, for reducing patient discomfort, which strongly supports preceding study.

\section{Acknowledgements}

We would like to thank all staff members from the departments of Radiology, Oral Radiology, Oral Surgery, Anesthesiology, Nursing, Dental Hygiene, and the clerks for their assistance in this study.

\section{Disclosure}

Authors report no conflict of interest.

\section{References}

1. Pernot M, Malissard L, Hoffstetter S et al. The study of tumoral, radiobiological, and general health factors that influence results and complications in a series of 448 oral tongue carcinomas treated exclusively by irradiation. Int J Radiat Oncol Biol Phys 1994; 29: 673-679.

2. Fein DA, Mendenhall WM, Parsons JT et al. Carcinoma of the oral tongue: a comparisbbb on of results and complications of treatment with radiotherapy and/or surgery. Head Neck 1994; 16: 358-365.

3. Shibuya H, Hoshina M, Takeda M et al. Brachytherapy for stage I \& II oral tongue cancer: an analysis of past cases focusing on control and complications. Int J Radiat Oncol Biol Phys 1993; 326: 351-358.

4. Hareyama M, Nishio M, Saito A et al. Results of cesium needle interstitial implantation for carcinoma of the oral tongue. Int J Radiat Oncol Biol Phys 1993; 25: 29-34. 
5. Mazeron JJ, Crook JM, Benck V et al. Iridium 192 implantation of T1 and T2 carcinomas of the mobile tongue. Int J Radiat Oncol Biol Phys 1990; 19: 1369-1376.

6. Inoue $\mathrm{T}$, Inoue $\mathrm{T}$, Yoshida $\mathrm{K}$ et al. Phase III trial of highvs. low-dose-rate interstitial radiotherapy for early mobile tongue cancer. Int J Radiat Oncol Biol Phys 2001; 51: 171-175.

7. Inoue $\mathrm{T}$, Inoue T, Teshima $\mathrm{T}$ et al. Phase III trial of high and low dose rate interstitial radiotherapy for early oral tongue cancer. Int J Radiat Oncol Biol Phys 1996; 36: 1201-1204.

8. Kakimoto $\mathrm{N}$, Inoue $\mathrm{T}$, Inoue $\mathrm{T}$ et al. Results of low- and highdose-rate interstitial brachytherapy for T3 mobile tongue cancer. Radiother Oncol 2003; 68: 123-128.

9. Yamazaki H, Inoue T, Yoshida K et al. Brachytherapy for early oral tongue cancer: low dose rate to high dose rate. J Radiat Res 2003; 44: 37-40.

10. Mazeron JJ, Ardiet JM, Haie-Meder C et al. GEC-ESTRO recommendations for brachytherapy for head and neck squamous cell carcinomas. Radiother Oncol 2009; 91: 150-156.

11. Nag S, Cano ER, Demanes DJ et al. The American Brachytherapy Society recommendations for high-dose-rate brachytherapy for head-and-neck carcinoma. Int J Radiat Oncol Biol Phys 2001; 50: 1190-1198.

12. Okamoto T, Akiyama H, Gamoh $S$ et al. High-dose-rate interstitial brachytherapy for tongue cancer; a radiological and biological study from the perspective of late complications. J Osaka Dent Univ 2012; 46: 57-62.

13. Akiyama H, Yoshida $K$, Shimizutani $K$ et al. Dose reduction trial from $60 \mathrm{~Gy}$ in 10 fractions to $54 \mathrm{~Gy}$ in 9 fractions schedule in High-dose-rate interstitial brachytherapy for early oral tongue cancer. J Radiat Res 2012; 53: 722-726.

14. McMahon J, Devine JC, McCaul JA et al. Use of Lugol's iodine in the resection of oral and oropharyngeal squamous cell carcinoma. Br J Oral Maxillofac Surg 2010; 48: 84-87.

15. Petruzzi M, Lucchese A, Baldoni E, et al. Use of Lugol's iodine in oral cancer diagnosis: an overview. Oral Oncol 2010; 46: 811-813.

16. Umeda M, Shigeta $T$, Takahashi $H$, et al. Clinical evaluation of Lugol's iodine staining in the treatment of stage I-II squamous cell carcinoma of the tongue. Int J Oral Maxillofac Surg 2011; 40: 593-596.

17. Yoshida K, Shimizutani K, Tanaka E, et al. Ultrasonographic monitoring of high dose rate interstitial implant using template technique for oral tongue cancer. Radiat Med 1999; 17: 337-341.

18. Yoshida K, Nose T, Koizumi M et al. The usefulness of metal markers for CTV-based dose prescription in high-dose-rate interstitial brachytherapy. J Jpn Soc Ther Radiol Oncol 2002; 14: 253-260.

19. Lau HY, Hay JH, Flores AD et al. Seven fractions of twice daily high dose-rate brachytherapy for node-negative carcinoma of the mobile tongue results in loss of therapeutic ratio. Radiother Oncol 1996; 39: 15-18.

20. Teshima $\mathrm{T}$, Inoue $\mathrm{T}$, Ikeda $\mathrm{H}$ et al. Phase I/II study of highdose rate interstitial radiotherapy for head and neck cancer. Strahlenther Onkol 1992; 168: 617-621.

21. Inoue T, Inoue T. High-dose rate interstitial brachytherapy for mobile tongue cancer: Part 2. Phase III trial of HDR versus LDR interstitial brachytherapy for T1-T2N0 carcinoma of the mobile tongue. Jpn J Cancer Chemother 2000; 27: Suppl 2: S291-295.

22. Yamazaki $Y$, Inoue $\mathrm{T}$, Yoshida $\mathrm{K}$ et al. Lymph node metastasis of early oral tongue cancer after interstitial radiotherapy. Int J Radiat Oncol Biol Phys 2004; 58: 139-146.

23. Benk V, Mazeron JJ, Grimard et al. Comparison of curietherapy versus external irradiation combined with curietherapy in stage II squamous cell carcinomas of the mobile tongue. Radiother Oncol 1990; 18: 339-347.
24. Pernot M, Malissard L, Aletti P et al. Iridium-192 brachytherapy in the management of $147 \mathrm{~T} 2 \mathrm{~N} 0$ oral tongue carcinomas treated with irradiation alone: comparison of two treatment techniques. Radiother Oncol 1992; 23: 223-228.

25. Shimizutani $\mathrm{K}$, Koseki, Inoue $\mathrm{T}$ et al. Interstitial Brachytherapy for Carcinoma of the tongue Using microSelectron-HDR. Oral Radiology 2001; 17: 95-101.

26. Yoshida K, Yamazaki H, Takenaka T et al. A dose-volume analysis of magnetic resonance imaging-aided high-doserate image-based interstitial brachytherapy for uterine cervical cancer. Int J Radiat Oncol Biol Phys 2010; 77: 765-772.

27. Major T, Polgar Cs, Mangel L et al. CT based conformal brachytherapy treatment planning. Hungarian Oncology 2000; 44: 109-115.

28. Nishioka T, Homma A, Furuta Y et al. A novel approach to advanced carcinoma of the tongue: cases successfully treated with combination of superselective intra-arterial chemotherapy and external/high-dose-rate interstitial radiotherapy. Jpn J Clin Oncol 2006; 36: 822-826. 